\title{
DIAGNOSTIC VALUE OF INTRAVENOUS FLUORESCEIN STUDIES*
}

\author{
BY \\ W. M. HAINING \\ Department of Ophthalmology, the Royal Infirmary of Edinburgh
}

A METHOD of studying the normal retinal circulation using intravenous fluorescein with retinal photography was first described by Novotny and Alvis (1961). The technique was modified and developed by Dollery, Hodge, and Engel (1962) to demonstrate pathological change of the retinal vasculature in diabetes mellitus and hypertension. It has been my aim to examine the diagnostic value of this procedure, particularly in relation to pseudo-papilloedema and plerocephalic papilloedema. In this paper the applicability of fluorescein studies to pseudopapilloedema will be discussed.

Spurious swelling of the optic nerve head can often be differentiated from true papilloedema using binocular indirect ophthalmoscopy and contact lens slit-lamp examination. The optical effect of nuclear lens sclerosis and the existence of optic disc anomalies with hypermetropia, excess papillary glial tissue, or visible drusen of the nerve head, are pitfalls well known to the experienced clinician.

There remains, however, a number of cases in which, from the evidence of a single examination, a categorical opinion cannot be given. These patients have normal visual acuity and the appearance of the disc may be discovered in the course of routine examination or in the aetiological investigation of a complaint of headache. It is in these problem cases, where there is a question of occult intracranial neoplasm, that an unequivocal opinion is most required.

Thus, any method of excluding the presence of papilloedema would be of assistance, both to the ophthalmologist and to the neurosurgeon.

\section{Photographic Requirements}

\section{Material and Methods}

The camera set-up is basically as described by Novotny and Alvis (1961), using a Zeiss Nordenson fundal camera with Ilford filters, numbers 304 Blue (exciting), peak transmission $430 \mathrm{~m} \mu$ and 110 Yellow (selective), peak transmission $550 \mathrm{~m} \mu$, incorporated into the camera system. The camera is equipped with an electronic flash, recharging at three second intervals; the flash setting III is required to give optimal illumination. The film used is Ilford HPS developed in Ilford Microphen for 25 minutes at $24^{\circ} \mathrm{C}$. $\left(75^{\circ} \mathrm{F}\right.$.).

\section{Catheterization Procedures}

Intra-arterial injection of fluorescein undoubtedly gives the best photographic results, but this method is not without risk. Grainger (1965) described the main complications of arterial catheterization as being local haemorrhage, arterial thrombosis, aneurysm formation, and arterial dissection. Brachial artery catheterization can produce arterial spasm with disastrous distal limb

\footnotetext{
* Received for publication April 27, 1965.
} 
ischaemia, and use of the carotid system may result in cerebral infarction. My main concern has been to evolve a simple, safe technique which can be performed as a routine clinical procedure by the ophthalmologist. The venous approach has, therefore, been selected and, although the presence of valves makes venous catheterization slightly more difficult to perform and the bolus of dye entering the cranial circulation is more diffuse, there is virtually no risk to the patient. In practice, the concentration of fluorescein in the retinal circulation has been found to be quite sufficient to demonstrate extremely fine fundal detail.

For the reasons described, catheterization of the superior vena cava employing a modified Seldinger technique has been the method of choice. This has been carried out in all cases as an out-patient procedure with local anaesthesia. A simple explanation of the procedure is first given to the patient and no form of premedication has been found to be necessary.

The patient lies recumbent on a standard theatre trolley, with the arm outstretched at an angle of $45^{\circ}$ from the body on a suitable supporting side platform. As a first stage it is always advisable to check that the flexible nylon "wire" can be freely drawn through the catheter and through the bore of the modified Riley needle in its entire length. Having done this, both catheter and wire are placed in a bowl of heparinized saline and heparinized saline is flushed through the catheter. A sphygmomanometer cuff is inflated round the upper arm to a level midway between the systolic and diastolic pressures and remains inflated throughout the entire procedure. The median cubital vein is identified and its position marked with a sterile chinagraph pencil. A small amount of 2 per cent. xylocaine is used to infiltrate the skin overlying the site of the venepuncture so as not to obscure the general position of the vein. The bared Riley needle is then inserted into the median cubital vein and, as soon as the lumen of the vein is completely entered by the bevel of the needle, the stilette is inserted and both needle and stilette are further advanced gently into the vein. It is important to state at this stage that the sphygmomanometer cuff should not be released, in order to maintain maximum patency of the venous system. The stilette is now withdrawn from the needle, when a free flow of blood should be obtained, and the solid nylon wire inserted into the needie lumen and advanced centrally without pressure. Using the point of a Number 15 blade mounted on a Bard-Parker handle, a small nick is made in the skin at the point of entrance of the needle to facilitate the subsequent insertion of a catheter. It is now necessary to withdraw the needle and, it must be stressed, the needle and nylon wire are together withdrawn as a unit until the needle point is clear from the arm. This avoids any chance of the sharp bevel of the needle slicing through the nylon wire whilst still within the lumen of the vein, and possible loss of the proximal section of the wire into the venous system. The nylon catheter is then threaded over the free end of the wire and advanced, using the wire as a rigid guide, until it has passed into the vein for some $20-30 \mathrm{~cm}$. A little patience may sometimes be required to insinuate the tip into the lumen of the vein. The central wire is withdrawn from the lumen of the catheter and a two-way disposable tap inserted with the spigot closed. The catheter can then readily be advanced into the region of the superior vena cava. This site is usually reached when approximately $10-15 \mathrm{~cm}$. of the catheter remains extra-vascular, but will obviously vary with the size and weight of the patient. The sphygmomanometer cuff can now be disinflated, the spigot turned open and the absence of clotting in the lumen of the catheter is ensured by gentle suction, using a syringe filled with heparinized saline. The catheter is then flushed and filled with heparinized saline and the tap closed. The catheter is taped to the patient's arm, he is allowed to walk through to the camera room and is seated in front of the camera. Preliminary straight photographs having been taken, a test dose of $0.5 \mathrm{ml}$. of a 5 per cent. solution of buffered sodium fluorescein is injected and a period of one minute is allowed to elapse to exclude the possibility of hypersensitivity to the dye. A further $5 \mathrm{ml}$. of the dye is then injected fairly rapidly. The first exposure is taken as soon as the first flush of fluorescein is seen in the camera viewing piece and serial photographs are subsequently taken (Figs 1 and 2, opposite). In plethoric, overweight patients twice the usual dose of fluorescein may be required to achieve adequate concentration in the retinal circulation. Finally, the catheter is withdrawn from the arm, steady pressure is exerted over the puncture site, and a small adhesive dressing is applied.

\section{Case Reports}

Case 1, a woman aged 20, was referred to the Medical Out-patients Department with a history of epigastric discomfort of the dyspeptic type for 5 months, associated with vomiting after meals. There was no complaint of headache or of any visual disturbance. 


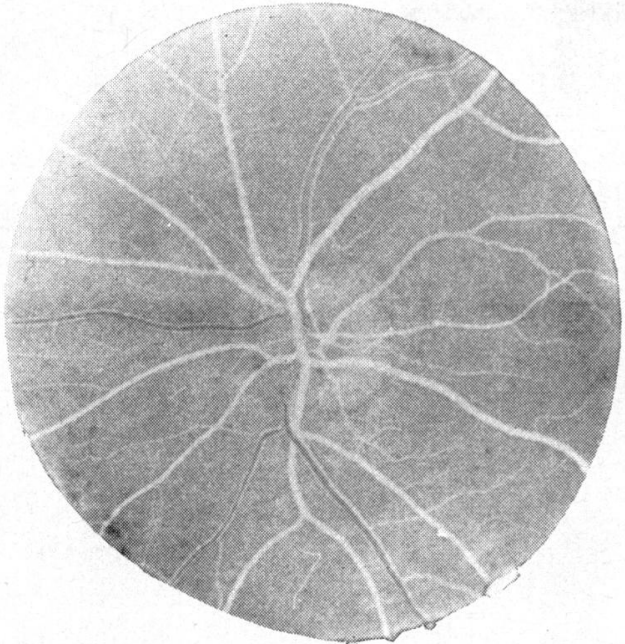

FIG. 1.-Normal optic disc, early venous filling phase.

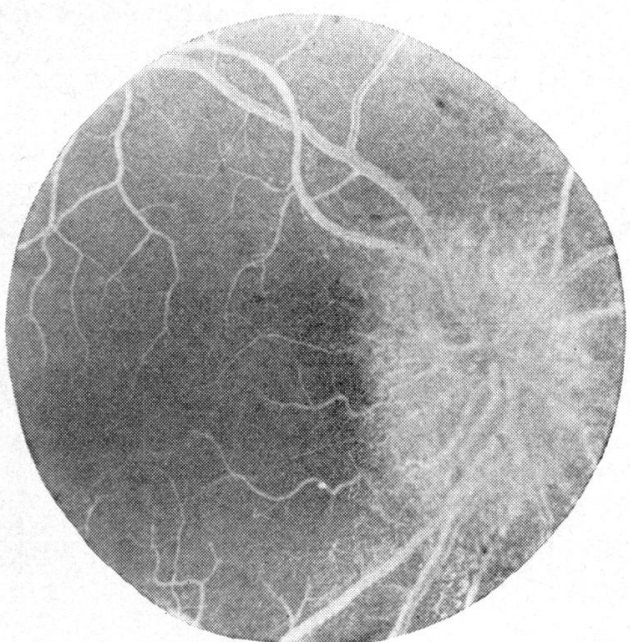

FIG. 2.-Moderate plerocephalic papilloedema without haemorrhages in a patient suffering from raised intra-cranial pressure.

General Examination.-Palpation revealed some epigastric tenderness and a barium meal showed the presence of duodenal deformity with a posterior ulcer crater to explain her symptoms. Routine examination of the central nervous system produced no positive findings, but the appearance of both optic discs was very suggestive of papilloedema, particularly in the left eye. Skull $X$-rays were reported to be normal. The patient was referred for an ophthalmic opinion before embarking upon any neurosurgical investigations.

Eye Examination.-There was no refractive error, the visual acuity was normal, and there was no significant loss of the visual field. Intravenous fluorescein studies were carried out and the results for the left eye are shown in Fig. $3 a, b$.

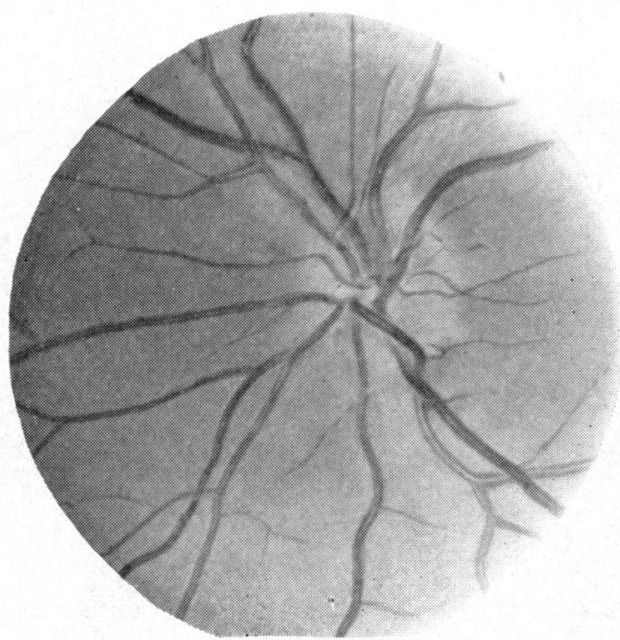

(a)

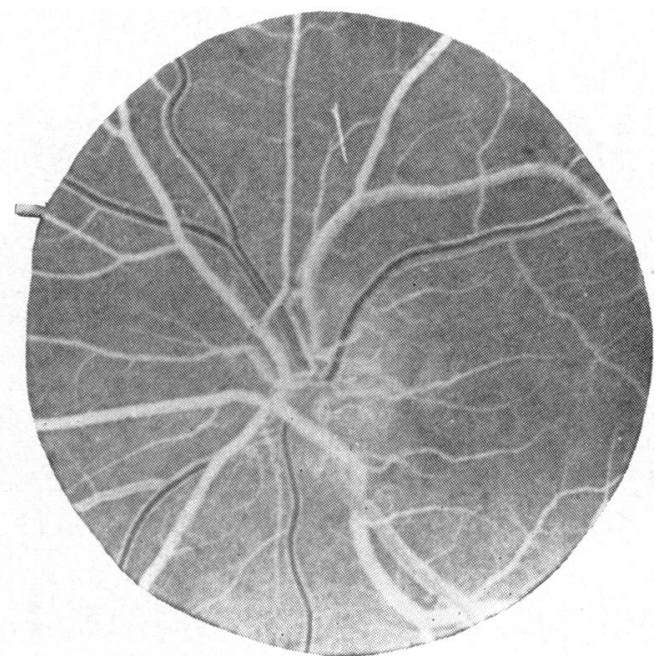

(b)

FIG. 3.-Case 1, left eye, (a) straight photograph; (b) early venous filling phase.

Comment.-The optic nerve head showed the faint fluorescent flush strictly delimited by the disc margins which is associated with the appearance of a "normal" optic disc. The apparent swelling could be ascribed to an excess of glial tissue.

Case 2, a girl aged 17, attended the Eye Clinic complaining of intermittent frontal headache of varying intensity. There were no other symptoms referable to the central nervous system. 
General Examination.-The patient was of slender build and somewhat anaemic, but otherwise looked well. There was no evidence of any abnormality of the cardiovascular system and the blood pressure was not raised. No abnormal signs were elicited in the central nervous system.

Eye Examination.-The visual acuity was normal with no refractive error, and the central fields of vision were unimpaired. The appearance of the optic discs was so suggestive of papilloedema that intravenous fluorescein studies were carried out to confirm this observation. The results in the left eye are shown in Fig. $4 a, b$.

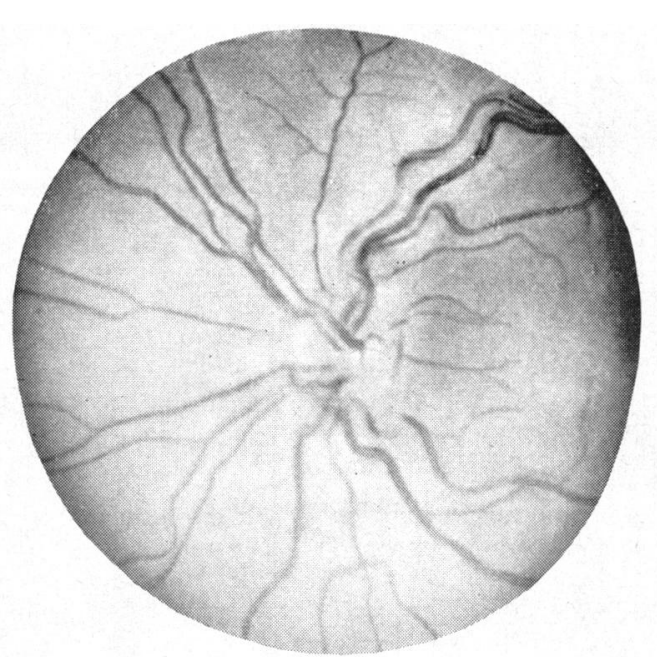

(a)

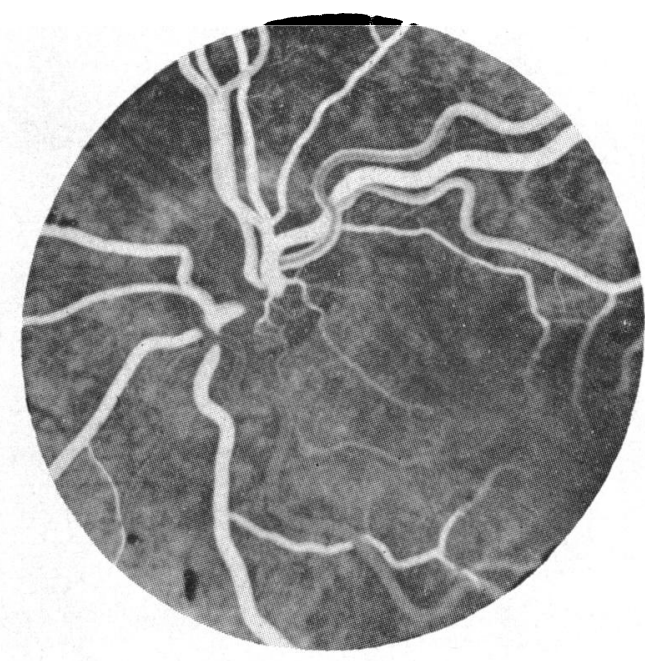

(b)

FIG. 4.-Case 2, left eye, (a) straight photograph; $(b)$ early venous filling phase.

Comment.-The optic nerve heads both showed an appearance similar to that of the previous case, excluding any suggestion of papilloedema.

Case 3, a woman aged 48, was admitted to hospital through the Casualty Department, having had an epileptiform convulsion followed by disordered behaviour. There was no past history of visual complaint or headache.

General Examination.-Neurological examination showed no clear signs, but it was noted that papilloedema was present in the right eye. The other positive findings were hepatic enlargement, an excessive urinary uribilinogen, and a raised serum bilirubin. At this point a tentative diagnosis was made of probable malignancy with involvement of the liver and brain. The patient was referred for neurosurgical opinion and, in view of the papilloedema, angiography was carried out with normal results. Within a further 24 hours the patient had recovered completely and her behaviour had returned to normal. The appearance of the optic disc, however, remained unchanged and she was referred for an ophthalmic opinion.

Eye Examination.-The visual acuity was normal with no refractive error, and the central field examination revealed no abnormality. The right optic disc appeared swollen, but the left was within normal limits. Fluorescein studies were done and the findings for the right eye are shown in Fig. $5 a, b$.

It was subsequently discovered that the probable aetiology of this case was related to an acute alcoholic state immediately before the patient's admission to hospital and that there was a past history of alcoholism.

Comment.-There was no evidence of any true papilloedema and, in view of the constancy of the optic disc appearance, it was unlikely that any oedema was previously present.

\section{Summary and Conclusions}

A simple method of fluorescein photography has been evolved to aid in the differential diagnosis of pseudopapilloedema. The catheterization technique, although more complex, is usually no more difficult to perform than a routine venepuncture. The illustrative cases presented suggest that this technique is of 
